\title{
CONHECIMENTO DOS PROFISSIONAIS DE ENFERMAGEM SOBRE ÚLCERAS POR PRESSÃO EM DUAS UNIDADES CIRÚRGICAS - PARTE 1
}

Nathalia Ingrid Crosewski', Débora de Sousa Lemos², Aline Batista Mauricio², Hellen Roehrs³, Marineli Joaquim Meier ${ }^{4}$

'Discente de Medicina. Universidade Federal do Paraná. Curitiba-PR-Brasil.

${ }^{2}$ Discente de Enfermagem. Universidade Federal do Paraná. Curitiba-PR-Brasil.

${ }^{3}$ Enfermeira. Doutoranda em Enfermagem. Docente da Universidade Federal do Paraná. Curitiba-PR-Brasil.

${ }^{4}$ Enfermeira. Doutora em Enfermagem. Docente da Universidade Federal do Paraná. Curitiba-PR-Brasil.

RESUMO: Realizou-se um estudo de caso em um hospital de ensino, no período de dezembro de 2012 a fevereiro de 2013, com objetivo de identificar o conhecimento dos profissionais de enfermagem sobre as úlceras por pressão em duas unidades cirúrgicas, com destaque para aspectos relativos à prevenção. Participaram 25 profissionais de enfermagem e utilizou-se um instrumento validado que considera conhecimento adequado aos que obtiverem $90 \%$ de acertos ou mais. A média de acertos das unidades foi igual a $73,88 \%$. Os erros mais comuns: o conceito de estágio III das úlceras por pressão e a indicação de massagem em regiões de proeminência óssea, elevação da cama a um ângulo superior de $30^{\circ}$, uso de luvas d'água, almofadas tipo roda d'água, reposicionamento de pacientes sentados. Os resultados evidenciam a necessidade de atualização sobre o tema úlceras por pressão de acordo com os guidelines.

DESCRITORES: Cuidados de enfermagem; Assistência à saúde; Úlceras por pressão.

\section{KNOWLEDGE OF NURSING PROFESSIONALS REGARDING PRESSURE ULCERS IN TWO SURGICAL UNITS - PART 1}

\begin{abstract}
A case study was undertaken in a teaching hospital, in December 2012 - February 2013, aiming to identify the knowledge of the nursing professionals regarding pressure ulcers in two surgical units, with emphasis on aspects relating to prevention. A total of 25 nursing professionals participated, and a validated instrument was used which considers appropriate knowledge to be held by those who obtained $90 \%$ of correct answers or more. The mean for correct answers in the units was equal to $73.88 \%$. The most common errors were: the concept of stage III of pressure ulcers and the indication of massage in regions of bony prominence, raising the bed to an angle greater than $30^{\circ}$, the use of gloves full of water, gel inflatable ring cushion, and the repositioning of seated patients. There is also evidence for the need for updating regarding the issue of pressure ulcers in accordance with the guidelines.
\end{abstract}

DESCRIPTORS: Nursing care; Healthcare; Pressure ulcers.

\section{CONOCIMIENTO DE LOS PROFESIONALES DE ENFERMERÍA SOBRE ÚLCERAS POR PRESIÓN EN DOS UNIDADES QUIRÚRGICAS - PARTE 1}

RESUMEN: Es un estudio de caso realizado en un hospital de enseñanza, en el periodo de diciembre de 2012 a febrero de 2013, con objetivo de identificar el conocimiento de los profesionales de enfermería acerca de las úlceras por presión en dos unidades quirúrgicas, con destaque para aspectos referentes a la prevención. Participaron 25 profesionales de enfermería y se utilizó un instrumento validado que considera conocimiento adecuado a los que obtuvieron $90 \%$ de aciertos o más. La media de aciertos de las unidades fue igual a $73,88 \%$. Los errores más comunes: el concepto de fase III de las úlceras por presión y la indicación de masaje en regiones de proeminencia ósea, elevación de la cama a un ángulo superior a $30^{\circ}$, uso de guantes d'água, almohadas redondas, reposicionamiento de pacientes sentados. Los resultados apuntan la necesidad de actualización sobre el tema úlceras por presión de acuerdo con los guidelines. DESCRIPTORES: Cuidados de enfermería; Asistencia a la salud; Úlceras por presión. 


\section{INTRODUÇÃO}

As úlceras por pressão (UP) são caracterizadas como lesões na pele que ocorrem, principalmente, em regiões de proeminência óssea, devido à pressão, cisalhamento e fricção. A pressão provoca falta de fluxo sanguíneo na região, causando isquemia e, consequente, necrose tecidual $^{(1-2)}$.

Em pacientes cirúrgicos as UP estão relacionadas ao tempo de cirurgia, posicionamento, sedação e umidade acentuada da pele pelo uso de produtos de antissepsia. Estima-se que a incidência de lesões ocasionadas pelo posicionamento cirúrgico inadequado varia entre $12 \%$ e $45 \%^{(3)}$.

Os cuidados para a prevenção e monitoramento de UP são de responsabilidade multiprofissional, entretanto a equipe de enfermagem é essencial na assistência a pacientes com essas feridas ${ }^{(4)}$. Assim, o conhecimento dos fatores para ocorrência de UP é fundamental, uma vez que compreendendo a causa é possível detectar pacientes propensos ao surgimento das lesões e direcionar ações para a prevenção( ${ }^{(5-7)}$, a fim de minimizar as consequências das UP.

A UP causa uma sobrecarga no trabalho dos profissionais da saúde, aumenta o uso de recursos materiais e os custos da internação(6). Aos pacientes, além do desconforto, essas lesões prolongam o tempo de internação, elevam o risco de desenvolvimento de infecções e consequente complicação no estado de saúde do paciente ${ }^{(8)}$.

Diante deste contexto é relevante avaliar o conhecimento dos profissionais a fim de identificar as possíveis lacunas na habilidade e competência sobre UP, para elaborar medidas educativas eficientes, suprir as deficiências e melhorar a qualidade do cuidado.

Uma das formas de avaliar o conhecimento dos profissionais de enfermagem foi desenvolvido por enfermeiras norte-americanas ${ }^{(9)}$ em formulário composto por 47 questões sobre prevenção, tratamento e monitoramento de UP. Este teste foi traduzido e adaptado à realidade brasileira ${ }^{(10-11)}$, na qual foram excluídas seis questões que referiamse às tecnologias não disponíveis no Brasil.

Compreendendo-se a importância do conhecimento na atuação do profissional de saúde para a qualidade do cuidado, pretende-se com esta pesquisa identificar o conhecimento que os profissionais possuem sobre UP, com enfoque na prevenção, em unidades Cirurgia Geral e Cirurgia do Aparelho Digestivo de um hospital de ensino.

\section{MÉTODO}

Realizou-se estudo de caso em dois serviços de enfermagem. A coleta de dados ocorreu de dezembro de 2012 a fevereiro de 2013, nos setores: Cirurgia Geral (CG) e Cirurgia do Aparelho Digestivo (CAD) de um hospital universitário de nível terciário de Curitiba, Paraná.

Os critérios de inclusão foram: ser profissional de enfermagem de nível médio ou graduado com mais de seis meses de experiência e não estar de licença no período da coleta. Como critério de exclusão foi a não entrega do formulário.

A unidade de internação CG possuía um total de 17 profissionais de enfermagem, dos integrantes do setor apenas 13 estiveram envolvidos na pesquisa: cinco enfermeiros, quatro técnicos e quatro auxiliares, pois um estava em férias e três recusaram-se a integrar ao estudo. $\mathrm{O}$ setor de CAD era composto por 17 profissionais sendo que 12 responderam o formulário: um enfermeiro, seis técnicos e cinco auxiliares de enfermagem, um estava em férias e quatro não participaram.

Para mensuração do conhecimento dos profissionais utilizou-se o formulário avaliativo elaborado por norte-americanos adaptado à realidade brasileira, indicado para todas as categorias profissionais de enfermagem, inclusive os estudantes. O instrumento é composto de perguntas sociodemográficas e 41 itens, assinalados com verdadeiro (V), falso ( F) ou não sei (NS), referentes aos conceitos e aos cuidados para a identificação, classificação e prevenção das UP. O conhecimento considerado adequado corresponde a $37(90 \%)$ acertos ou mais $^{(11)}$.

Os dados foram tabulados com auxilio do programa Microsoft Office Exce/2007. As questões respondidas corretamente corresponderam a um ponto e as incorretas ou não respondidas não pontuaram. Considerou-se o conhecimento dos profissionais satisfatório quando a soma de pontos no teste correspondeu a 37 pontos ou mais de $90 \%$ de acerto. 
O projeto foi aprovado pelo Comitê de Ética do Hospital de realização da pesquisa, número de parecer 181.924 em 26 de dezembro de 2012. Os princípios éticos foram respeitados conforme a Resolução 466/12, após assinatura do Termo de Consentimento Livre-Esclarecido.

\section{RESULTADOS}

Para a contextualização do cenário sociodemográfico dos participantes apresentamos os seguintes resultados: dos 17 profissionais de enfermagem do setor CG, 13 (76,47\%) participaram, 12 (92,31\%) correspondiam ao sexo feminino, a idade média foi de 44,33 anos e seis $(46,15 \%)$ disseram frequentar cursos e palestras sobre UP. No setor CAD dos 17 profissionais, $12(70,59 \%)$ responderam ao questionário, oito
$(66,67 \%)$ eram do sexo feminino, a idade média foi de 46,83 anos e apenas três (25\%) afirmaram participar de atividades educativas sobre o tema.

Os resultados individuais dos setores mostram média de acertos de 32,08 (78,24\%) no setor CG e 28,50 (69,51\%) no serviço da CAD. Destaca-se a diferença de 8,73 pontos percentuais entre as médias de acertos das unidades. Nenhuma das unidades atingiu a pontuação considerada como satisfatória que seria de 37 acertos ou mais, pois a média das duas unidades foi de 30,29 (73,88\%) acertos.

Ao todo, na CG nenhum dos avaliados alcançou os $90 \%$ ou mais de acertos considerados necessários, apesar de 53,85\% dos profissionais acertarem mais de $80 \%$ das questões. O setor CAD obteve um desempenho inferior ao da CG, como representado na Tabela 1.

Tabela 1 - Distribuição dos participantes no teste de conhecimento sobre UP, segundo a porcentagem de acertos. Curitiba-PR, 2013

\begin{tabular}{lccc}
\hline \multirow{2}{*}{ Porcentagem de acertos } & Cirurgia Geral $(\mathbf{n}=\mathbf{1 3})$ & $\begin{array}{l}\text { Cirurgia do Aparelho } \\
\text { Digestivo }(\mathbf{n}=\mathbf{1 2})\end{array}$ & Total $(\mathbf{n}=\mathbf{2 5})$ \\
\cline { 2 - 4 } & $\mathbf{F}$ & $\mathbf{F}$ & $\mathbf{F}$ \\
\hline 50 à 60 & 01 & 01 & 02 \\
\hline 60 à 70 & 01 & 07 & 08 \\
\hline 70 à 80 & 04 & 02 & 06 \\
\hline 80 à 90 & 07 & 02 & 09 \\
\hline$\geq 90$ & 0 & 0 & 0 \\
\hline Total & 13 & 12 & 25 \\
\hline
\end{tabular}

Legenda: V - verdadeiro; F - falso; CG - Cirurgia Geral; CAD - Cirurgia do Aparelho Digestivo.

Quanto aos oito itens sobre conceitos de úlcera por pressão e estadiamento, a unidade CG mostrou falta de uniformidade no conhecimento. Apesar dos avaliados obterem $100 \%$ de acerto em duas questões sobre estadiamento, observou-se que em outras afirmações os acertos foram de $53,85 \%$ e $61,54 \%$. A média na porcentagem de acertos dessa categoria foi de $75,96 \%$. Na CAD a média na porcentagem de acertos nesta categoria foi de $48,96 \%$ o que corresponde a 27,01 pontos percentuais a menos que a média do outro setor. Também, observou-se a mesma discrepância de conhecimentos. Uma vez que em uma das questões o número de acerto foi de $0 \%$.

Nos seis itens relacionados aos fatores de risco, os profissionais de enfermagem do setor CG mostraram conhecimento satisfatório, ou seja, acertaram mais de $90 \%$ exceto pela questão sobre higiene da pele, como o uso de sabonetes e água quente, o que resultou em uma média de $89,75 \%$ acertos. O setor CAD obteve um resultado muito parecido e com um baixo desempenho na questão sobre a higiene da pele. A média da porcentagem de acertos do setor foi igual a $86,11 \%$. Na totalidade da categoria as unidades obtiveram média de $88 \%$, sendo que a diferença entre as médias dos setores foi de 3,63 pontos percentuais.

O setor CG obteve desempenho satisfatório em relação às 13 questões de prevenção, a citar: inspeção de pele, umidade/incontinências, nutrição e avaliação de risco. A média foi de $94,68 \%$, sendo que em oito questões a equipe obteve $100 \%$ de acertos. O setor CAD obteve uma média de 91,03\% de acertos. Destaca-se que do total de questões sobre prevenção da UP os profissionais acertaram $100 \%$ em seis 
delas. Quanto ao resultado total obtido pelas duas unidades, obteve-se média de acertos igual 89,54\%. A diferença entre as médias dos setores foi de 3,65 pontos percentuais.

No quesito medidas de prevenção dos fatores extrínsecos como: forças de fricção, cisalhamento e pressão e mobilidade do paciente totalizaram 14 questões. O setor CG obteve 100\% de acerto em três questões relativas à mobilidade do paciente. A média de porcentagem no bloco foi igual a 59,34\%. O setor CAD obteve a média de porcentagem de acerto de 54,17\%. Das 14 questões, os profissionais acertaram 100\% de duas delas relativas à mobilidade do paciente. $\mathrm{A}$ média geral obtida pelas duas unidades foi igual a $53,71 \%$, e a diferença entre as médias das duas unidades foi de 5,17 pontos percentuais. Os acertos estão descritos na tabela 2.

Tabela 2 - Porcentagem de acertos no formulário avaliativo de medidas de prevenção da UP em unidades de cirurgia geral e cirurgia do aparelho digestivo. Curitiba-PR, 2013

\begin{tabular}{|c|c|c|c|c|c|c|}
\hline \multirow{2}{*}{ Questões } & \multicolumn{2}{|c|}{ CG $(n=13)$} & \multicolumn{2}{|c|}{ CAD $(n=12)$} & \multicolumn{2}{|c|}{ Total $(n=25)$} \\
\hline & $f$ & $\%$ & $f$ & $\%$ & $f$ & $\%$ \\
\hline $\begin{array}{lccc}\text { É importante massagear as regiões das } \\
\text { proeminênciasósseas, seestiveremhiperemiadas }(F) \text {. }\end{array}$ & 0 & 0 & 02 & 16,67 & 02 & 08 \\
\hline $\begin{array}{l}\text { Os cremes, curativos transparentes e curativos de } \\
\text { hidrocoloides extrafinos auxiliam na proteção da } \\
\text { pele contra os efeitos da fricção (V). }\end{array}$ & 12 & 92,31 & 11 & 91,67 & 23 & 92 \\
\hline $\begin{array}{l}\text { Os pacientes que ficam restritos ao leito devem } \\
\text { ser reposicionados a cada } 3 \text { horas (F). }\end{array}$ & 10 & 76,92 & 06 & 50 & 16 & 64 \\
\hline $\begin{array}{l}\text { Escala com horários para mudança de decúbito deve ser } \\
\text { usada em pacientes com presença ou risco para UP }(\mathrm{V}) \text {. }\end{array}$ & 9 & 69,23 & 11 & 91,67 & 20 & 80 \\
\hline $\begin{array}{l}\text { Luvas d'água ou de ar aliviam a pressão nos } \\
\text { calcâneos (F). }\end{array}$ & 4 & 30,77 & 0 & 0 & 4 & 16 \\
\hline $\begin{array}{l}\text { As almofadas tipo rodas d'água ou de ar auxiliam } \\
\text { na prevenção da UP (F). }\end{array}$ & 4 & 30,77 & 0 & 0 & 4 & 16 \\
\hline $\begin{array}{l}\text { Na posição em decúbito lateral, o paciente } \\
\text { com presença da UP ou em risco para a mesma } \\
\text { deve ficar em ângulo de } 30 \text { graus em relação ao } \\
\text { colchão do leito }(V) \text {. }\end{array}$ & 4 & 30,77 & 4 & 33,33 & 8 & 32 \\
\hline $\begin{array}{l}\text { No paciente com presença da UP ou em risco } \\
\text { para a mesma, a cabeceira da cama não deve ser } \\
\text { elevada em ângulo maior do que } 30 \text { graus, se não } \\
\text { houver contraindicação médica }(\mathrm{V}) \text {. }\end{array}$ & 0 & 0 & 4 & 33,33 & 4 & 16 \\
\hline $\begin{array}{l}\text { Paciente que não se movimenta sozinho deve ser } \\
\text { reposicionadoacada } 2 \mathrm{~h} \text {, quando sentado na cadeira }(\mathrm{F}) \text {. }\end{array}$ & 04 & 30,77 & 0 & 0 & 04 & 16 \\
\hline $\begin{array}{l}\text { O paciente com mobilidade limitada e que pode } \\
\text { mudar a posição do corpo sem ajuda deve ser } \\
\text { orientado a realizar o alívio da pressão, a cada } 15 \\
\text { minutos, enquanto estiver sentado na cadeira (V). }\end{array}$ & 11 & 84,62 & 07 & 58,33 & 18 & 72 \\
\hline $\begin{array}{l}\text { O paciente com mobilidade limitada e que } \\
\text { pode permanecer na cadeira, deve ter uma } \\
\text { almofada no assento para proteção da região das } \\
\text { proeminências ósseas }(V) \text {. }\end{array}$ & 13 & 100 & 11 & 91,67 & 24 & 96 \\
\hline $\begin{array}{l}\text { Os lençóis móveis ou forros devem ser utilizados } \\
\text { para transferir ou movimentar pacientes que não } \\
\text { se movimentam sozinhos }(\mathrm{V}) \text {. }\end{array}$ & 13 & 100 & 12 & 100 & 25 & 100 \\
\hline $\begin{array}{l}\text { A mobilização e a transferência de pacientes que } \\
\text { não se movimentam sozinhos devem ser sempre } \\
\text { realizadas por duas ou mais pessoas }(\mathrm{V}) \text {. }\end{array}$ & 13 & 100 & 12 & 100 & 25 & 100 \\
\hline $\begin{array}{l}\text { Uma boa maneira de diminuir a pressão na região } \\
\text { dos calcâneos é mantê-los elevados do leito (V). }\end{array}$ & 11 & 84,62 & 11 & 91,67 & 22 & 88 \\
\hline
\end{tabular}

Legenda: V - verdadeiro; F - falso; CG - Cirurgia Geral; CAD - Cirurgia do Aparelho Digestivo. 


\section{DISCUSSÃO}

A média de $73,88 \%$ de acertos obtidos pelos setores se assemelha aos $73,5 \%$ de acertos conseguidos por 50 profissionais de enfermagem em um Centro de Terapia Intensiva de um hospital terciário do interior de São Paulo ${ }^{(12)}$. Ainda, pode-se citar uma pesquisa realizada no Hospital Universitário Sul Fluminense do Rio de Janeiro, quando 51 profissionais alcançaram média de $71,5 \%$, Estes trabalhavam nas unidades na UTI adulto e neonatal, no pronto socorro, nos apartamentos de convênios e particulares, nas enfermarias adultas e pediátricas ${ }^{(13)}$.

Diante desse resultado, observa-se a necessidade de atualização constante da equipe de enfermagem quanto aos cuidados necessários para a prevenção e tratamento da UP, uma vez que não alcançaram a porcentagem necessária para ter um conhecimento satisfatório.

Os participantes demonstraram conhecimento satisfatório em alguns quesitos como o estadiamento da UP em grau I e IV. Essa informação é necessária para iniciar o tratamento precocemente, uma vez que a UP no estágio I, ou seja, hiperemia e pele íntegra, com medidas de cuidados adequadas, como alivio da pressão, a cicatrização pode ocorrer 24 horas $^{(14)}$. Portanto, avaliar a lesão, as características e indicar as ações adequadas são conhecimentos imprescindíveis ao enfermeiro, desde o início da sua formação acadêmica.

Nas unidades cirúrgicas as UP em estágio I são comuns, uma vez a permanência de duas horas ou mais do paciente em posicionamento cirúrgico já predispõem ao aparecimento de UP(15). Outro aspecto relevante no paciente cirúrgico é que a dor presente no pós-operatório pode diminuir a mobilidade física e o autocuidado ${ }^{(16)}$, propiciando o surgimento de UP.

Em pacientes com obesidade mórbida que realizam cirurgia bariátrica, as principais complicações estão relacionadas com a imobilização do paciente. Entre essas complicações estão as úlceras por pressão ${ }^{(17)}$.

Dos erros cometidos pelos profissionais destacam-se as questões de higienização da pele, massagem em regiões de proeminência óssea, elevação da cama a um ângulo superior de $30^{\circ}$, uso de luvas d'água, almofadas tipo roda d'água e sabão e reposicionamento de pacientes.
Com relação à higienização da pele salienta-se que a água quente e o sabonete podem ressecar a pele e deixá-la predisposta a ruptura. O banho com sabonete desencadeia um aumento do $\mathrm{pH}$ da pele, que interfere na proteção fisiológica dessa barreira, provocando mudança na composição da microbiota cutânea. $\mathrm{O}$ uso desse produto também pode afetar a epiderme ao dissolver a gordura da superfície desta e alterar a atividade de enzimas. Com isso, a hidratação é prejudicada e há predisposição para secura e a descamação da pele ${ }^{(18)}$.

A massagem em regiões de proeminência óssea quando a pele está hiperemiada não é indicada para prevenção de UP. Acreditando que a massagem ajudará na oxigenação da área muitos profissionais utilizam essa prática, entretanto a atividade só estará acrescentando pressão, podendo causar mais danos ${ }^{(1,12,19)}$.

A cama não deve ser elevada a um ângulo superior a $30^{\circ}$ em casos de pacientes com UP ou em risco de adquiri-las, pois o deslizamento do paciente na cama ocasiona cisalhamento e como consequência ocorrem lesões na região sacral $^{(1,17,19)}$. Quanto ao reposicionamento em cadeira, este deve ser realizado no mínimo a cada hora quando o paciente não se movimenta sozinho, inclinando o acento para reduzir o contato com as nádegas do paciente e a superfície de apoio. Recomenda-se, também, o uso de almofadas de gel ou ar para alivio da pressão nas proeminências ósseas ${ }^{(14)}$.

Luvas e almofadas tipo roda d'água não devem ser usadas para o alivio da pressão, pois estas localizam a pressão, possibilitando assim o surgimento de lesões ${ }^{(2,20)}$. E ainda, as luvas ou almofadas de água em contato com a pele ou em contato direto com o pijama promovem vasoconstrição; favorecem o desenvolvimento de patologias devido à carência do aporte de oxigênio, como é o caso do desenvolvimento de UP(21).

A redistribuição mecânica do paciente é uma estratégia importante e deve ser feita de forma adequada para que surta efeito. Já estão disponíveis no mercado alguns colchões com espumas especiais de viscoelástico, que podem ser usadas nos centros cirúrgicos, pois possibilitam a prevenção da UP(15).

Em um estudo realizado em um hospital universitário do sul do Brasil, com 219 prontuários de pacientes com UP, observou-se que o cuidado de proteger proeminências ósseas foi o terceiro 
cuidado mais prescrito por enfermeiros ${ }^{(22)}$.

Alguns dos erros cometidos como luvas d'água, almofadas de roda e massagem ocorrem devido à desatualização dos funcionários. Essas medidas, que outrora eram recomendadas, continuam em uso, promovendo equívocos no atendimento $^{(5,7,12,23)}$. A educação continuada pode minimizar ou solucionar esses enganos, promovendo atualização do conhecimento científico ${ }^{(23-24)}$.

Devido ao pequeno número de participantes e a ausência de preenchimento de informação no formulário referente à atualização sobre UP, não foi possível correlacionar esses dados com a média de acertos do questionário. Existia a hipótese de que a participação em atividades educativas relacionadas à UP aumentaria a média de acerto. Esta assertiva foi verificada em um estudo, no qual a intervenção educacional aumentou a porcentagem de acerto de um grupo de auxiliares e técnicos de enfermagem de $74,3 \%$ para $81,2 \%$, confirmando a importância da atualização do conhecimento ${ }^{(13)}$.

Entretanto, alguns programas de educação continuada de instituições de saúde possuem limitada capacidade de produzir mudanças, pois mantêm a lógica programática das ações, não desafiando os participantes e nem problematizando suas próprias práticas. Os resultados da educação continuada acabam permanecendo somente no papel ou nas apostilas. Deste modo, não há mudanças significativas entre o antes e o depois do programa de educação continuada. Para garantir melhores resultados deve-se avaliar as lacunas de conhecimento existente, antes da realização de estudos e disseminação do conhecimento ${ }^{(24)}$.

\section{CONCLUSÕES}

O conhecimento é um requisito fundamental para as boas práticas em enfermagem. No caso das UP este conhecimento evita a dor e o sofrimento para pacientes, bem como redução de custos para as instituições. Foi evidenciado o déficit de conhecimento nas duas unidades pesquisadas em aspectos relacionados a prevenção das UP e ações que não são mais indicadas pelos guidelines internacionais.

Os resultados apontam para a necessidade e a importância da educação continuada para a atualização e o aprofundamento de conhecimentos para os profissionais em serviço, para atendimento de qualidade ao paciente.

\section{REFERÊNCIAS}

1. National Pressure Ulcer Advisory Panel [Internet] National Pressure Ulcer Advisory Panel, 2009. [acesso em 20 fev 2014]. Disponível: http://www.npuap.org/

2. White-Chu EF, Reddy M. Pressure ulcer prevention in patients with advanced illness. Curr Opin Support Palliat Care. 2013;7:111-5.

3. Barbosa MH, Ártemis AMBO, Neto ALS. Ocorrência de lesões perioperatórias por posicionamento cirúrgico. Rev Cubana Enferm. 2011;27(1):31-41.

4. Lucena ADF, Santos CT, Pereira AGS, Almeida MA, Dias VLM, Friedrich MA. Perfil clínico e diagnósticos de enfermagem de pacientes em risco para úlcera por pressão. Rev. Latino-Am. Enfermagem. 2011;19(3):1-8.

5. Araújo TM, Araújo MFM, Caetano JA, Galvão MTG, Damascenoi MMC. Diagnósticos de enfermagem para pacientes em risco de desenvolver úlcera por pressão. Rev. bras. enferm. 2011;64(4):671-6.

6. Pott FS, Ribas JD, Silva OBM, Souza TS, Danski MTR, Meier MG. Algoritmo de prevenção e tratamento de úlcera por pressão. Cogitare enferm. 2013; 18(2):238-44.

7. Figueiras RG. Tratamento cirúrgico de úlceras por pressão : experiência de dois anos. Rev. Bras. Cir. Plást. 2011;26(3):418-27.

8. Parra DL, Camargo-Figueira FA, Gómez RR. Eventos adversos derivados del cuidado de enfermería: flebitis, úlceras por presión y caídas. Enferm. glob. [Internet] 2012;11(4) [acesso em 23 mar 2013]. Disponível: http:// revistas.um.es/eglobal/article/view/eglobal.11.4.150551

9. Pieper B, Mott M. Nurses' knowledge of pressure ulcer prevention, stating, and description. Adv Wound Care. 1995;8(3):34-48.

10. Caliri MHL, Miyazaki MY, Piper B. Knowledge about pressure ulcers by undergraduate nursing students in Brazil. Ostomy Wound Manage. 2003;49(3):54-63.

11. Fernandes LM. Efeito de intervenções educativas no conhecimento e práticas de profissionais de enfermagem e na incidência de úlcera por pressão em centro de terapia Intensiva [tese]. Ribeirão Preto: Escola de Enfermagem de Ribeirão Preto - Universidade de São Paulo; 2006.

12. Fernandes LM, Caliri MHL, Haas VJ. Efeito de intervenções educativas no conhecimento dos profissionais de enfermagem sobre prevenção de 
úlceras pressão. Acta Paul. Enferm. 2008;21(2):305-11.

13. Fernandes NCN, Amarali JPBV. Conhecimento da equipe multidisciplinar sobre prevenção, avaliação e tratamento de úlcera de pressão no Hospital Universitário Sul Fluminense/RJ. Estação Científica - Edição Especial "Fisioterapia" [Internet] 2012:1(1) [acesso em 24 mar 2013]. Disponível: http://portal. estacio.br/media/3878443/conhecimento $\% 20 \mathrm{da} \% 20$ equipe\%20multisciplinar.pdf

14. Luz SR, Lopacinski AC, Fraga R, Urban CA. Úlceras de pressão. Geriatr. gerontol. 2010;4(1):36-43 [acesso em 26 jul 2014]. Disponível: http://www.sbgg.org. br/profissionais/arquivo/revista/volume4-numero1/ artigo06.pdf

15. Fonseca RMP, Peniche ACG. Enfermagem em centro cirúrgico: trinta anos após criação do Sistema de Assistência de Enfermagem Perioperatória. Acta Paul. Enferm. 2009;22(4):428-33.

16. Bertoncello KCG, Sávio B, Ferreira JM, Amante LN, Nascimento ERP. Diagnósticos e propostas de intervenções de enfermagem aos pacientes em pósoperatório imediato de cirurgia eletiva. Cogitare enferm. 2014;19(3):582-9.

17. Nibi FA, Osti C. Cuidados intensivos no pósoperatório imediato de cirurgia bariátrica. Rev. Uninga. 2014;(39):149-58.

18. Fontenele FC, Cardoso MVLML. Lesões de pele em recém-nascidos no ambiente hospitalar: tipo, tamanho e área afetada. Rev. Esc. Enferm. USP. 2011;45(1):130-7.

19. Miyazaki MY, Caliri MHL, Santos CB. Conhecimento dos profissionais de enfermagem sobre prevenção da úlcera por pressão. Rev. Latino-Am. Enfermagem [Internet] 2010;18(6) [acesso em 20 mar 2013]. Disponível: http://dx.doi.org/10.1590/S010411692010000600022

20. Rolim JA, Vasconcelos JMB, Caliri MHL, Santos IBC. Prevenção e tratamento de úlceras por pressão no cotidiano de enfermeiros Intensivistas. Rev Rene. 2013;14(1):148-57.

21. Rogenski N, Kurcgant P. Avaliação da concordância na aplicação da Escala de Braden. Acta Paul. Enferm. [Internet]. 2012;25(1) [acesso em 1 jul 2013] Disponível: http://www.scielo.br/pdf/ape/v25n1/v25n1a05.pdf

22. Pereira AGS, Santos CT, Menegon DB, Mello BS, Azambuja F, Lucena AF. Mapeamento de cuidados de enfermagem com a NIC para pacientes em risco de úlcera por pressão. Rev. Esc. Enferm. USP. 2014;48(3). Disponível: http://dx.doi.org/10.1590/ S0080-623420140000300010

23. Peixoto LS, Gonçalves CL, Costa TD, Tavares CMM, Cavalcanti ACD. Educação permanente, continuada e em serviço: desvendando seus conceitos. Enferm. glob. [Internet] 2013;12(1) [acesso em 25 mar 2013]. Disponível: http://revistas.um.es/eglobal/article/ view/141801

24. Silva MF, Conceição FA, Leite MMJ. Educação continuada: um levantamento de necessidades da equipe de enfermagem. Mundo saúde [Internet]. 2008;32(1) [acesso em 20 jan 2014]. Disponível: http:// www.saocamilo-sp.br/pdf/mundo_saude/58/47a55.pdf 